\title{
Long COVID braucht Rehabilitation
}

\author{
Nach einer Infektion mit dem SARS-CoV-2-Virus können Patienten wochen- \\ und monatelang von Folgesymptomen betroffen sein. Wichtig sind \\ entsprechende Maßnahmen zur Rehabilitation. \\ Christian F. Freisleben
}

Vergangenen Sommer verdichteten sich die Hinweise darauf, dass es ein „Long COVID“ gibt, Langzeitfolgen einer Corona-Infektion. „Hilfreich sind Erfahrungswerte der SARS-Pandemie 2002/2003 sowie Beobachtungen zum vor allem auf der arabischen Halbinsel auftretenden Middle East Respiratory Syndrome Coronavirus MERS“, erläutert Bernd Lamprecht, Generalsekretär der Österreichischen Gesellschaft für Pneumologie und Chef der Lungenheilkunde am Kepler Universitätsklinikum. Betroffen sein könnten vier Prozent der Personen, die sich mit dem neuartigen Coronavirus infiziert haben, wobei eine Schweizer Studie davon ausgeht, dass jeder dritte COVID-Patient mehrere Wochen Folgesymptome haben kann. ${ }^{1}$ „Es ist ein vielfältiges Bild an Beschwerden, die auftreten, wobei die Frage ist, was Patienten an sich beobachten und

Bernd Lamprecht, Kepler Universitätsklinikum:
"Aufmerksames Beobachten
und gezieltes Nachfragen."

was sie im Kontakt mit dem niedergelassenen Arzt oder Krankenhaus berichten. "Lamprecht betont, dass aufmerksames Beobachten und gezieltes Nachfragen entscheidend sind.

Im Vordergrund bei Long COVID steht Fatigue, nicht erklärbare Müdigkeit, Abgeschlagenheit und Antriebslosigkeit. Dazu kann Atemnot bei Belastungen kommen, auch bei Alltagstätigkeiten wie Treppensteigen oder Einkaufen. In Lungenfunktionsuntersuchungen kann ein Substrat nachgewiesen werden, die Fähigkeiten zum Gasaustausch und zur Sauerstoffaufnahme der Lunge sind noch nicht normalisiert - und das über mehrere Wochen hinweg. Häufig berichtet werden ebenso psychische Symptome, Ängstlichkeit, Schlafstörungen, depressive Verstimmung - „das sind Nachwirkungen eines Spitalsaufenthalts und Konse- quenzen davon, dass viele Probleme dabei haben, ihre Krankheit zu verarbeiten, einen Schlussstrich zu ziehen, in einer Zeit, wo es zur Pandemie stündlich Neuigkeiten in den Medien und Alltagsgesprächen gibt“, ergänzt Lamprecht.

\section{Früh Maßnahmen setzen}

Wichtig ist, Long COVID so früh wie möglich zu erkennen sowie schon während des primären Krankenhausaufenthalts Maßnahmen zu setzen. „Auch bei Personen mit intensiverem Krankheitsverlauf, die in häuslicher Betreuung sind, kann es zu Long COVID kommen." Lamprecht betont die Bedeutung von möglichst bald beginnender Bewegungstherapie, also Physiotherapie mit Fokus auf die Atemwege und den Bewegungsapparat. Nach einem Spitalsaufenthalt ist eine stationäre oder ambulante Anschlussrehabilitation

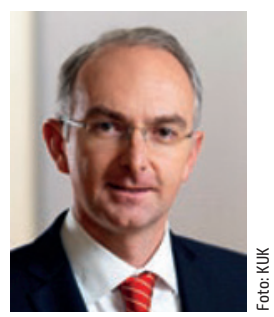
mit einem multidisziplinären Ansatz angezeigt: mit Beteiligung von Fachleuten aus Neurologie, Lungenheilkunde, Psychologie, Diätologie und Sportmedizin. „Danach sind Maßnahmen zur Unterstützung wichtig, damit Patienten das Gelernte im Alltag umsetzen können“, so Lamprecht. Er empfiehlt bei allen Angeboten zur Rehabilitation eine Ausrichtung auf die im Juni 2020 publizierten Empfehlungen der Deutschen Gesellschaft für Pneumologie ${ }^{2}$. Ebenso gibt es Empfehlungen aus Großbritannien. ${ }^{3}$

„Zwar gibt es Begriffe wie Long oder Post COVID bereits, der Findungsprozess für die optimalen Formen der Rehabilitation bei diesen Phänomenen, die noch genauer zu untersuchen sind, läuft aber noch." Ebenso werde laut Lamprecht diskutiert, ob der Begriff „Long“ tatsächlich gerechtfertigt ist. Im Vergleich zu SARS 1 seien jedenfalls dauerhafte Schädigungen der Lungen wesentlich seltener zu erwarten.

Lamprecht betont, dass von Long COVID vor allem ältere und gebrechliche Personen betroffen sind. „Es braucht ausreichende Kapazitäten an stationärer Rehabilitation, speziell auch für die- 
se Zielgruppe. Sehr wichtig ist eine hohe Sensibilität von Allgemeinmedizinern und niedergelassenen Fachärzten, auch in den zumindest nächsten drei bis vier Jahren." Keine Notwendigkeit sieht Lamprecht für die Einrichtung von Spezialambulanzen für Menschen mit Long COVID.

\section{Verschiedene Angebote in Österreich}

Das Herz-Jesu-Krankenhaus in Wien nimmt seit Oktober 2020 Patienten aus Wiener Spitälern auf, die nach einem schweren COVID-19-Erkrankungsverlauf intensiv rehabilitiert werden müssen. Für diese Akutrehabilitation wurde ein Team aus den Bereichen Pulmologie, Radiologie, Diätologie und Klinische Psychologie sowie Seelsorge zusammengestellt. Das Hauptziel besteht darin, die reduzierte Lungenfunktion zu verbessern bzw. uneingeschränkt wiederherzustellen. Neben Übungen zur Stärkung der Atemmuskelkraft und der muskulären Kräftigung sowie Mobilisierung der Patienten geht es um die Erhöhung der pulmonalen Belastbarkeit. Im Fokus sind aber auch psychische, neurologische, kardiale und muskuläre Beeinträchtigungen. Um die Rückkehr in den Alltag zu erleichtern, gibt es Unterstützung bei der Trauma-, Angst- und Depressionsbewältigung sowie Verbesserung der Ernährung.

Die III. Medizinische Abteilung für Innere Medizin und Psychosomatik am Barmherzige Schwestern Krankenhaus Wien stellte im Jänner ein Post-COVID-Programm vor: Die ärztliche Leiterin Larisa Dzirlo betont die Wichtigkeit, auch auf die psychischen Symptome zu blicken: Zu Angststörungen, Depressionen, Panikattacken oder posttraumatischem Stresssyndrom kann Todesangst kommen oder die Befürchtung, Überträger zu sein und andere Menschen zu gefährden. Dazu kommt die Erfahrung sozialer Isolation, Einsamkeit und Frustration. Das ambulante Programm findet über sechs Wochen zweimal pro Woche statt. Es besteht aus psychotherapeutischen Modulen sowie medizinischer Visite, körperlicher Betätigung (Training), Entspannung und Einzelpsychotherapie.

Roland Winkler, Ärztlicher Leiter am Rehabilitationszentrum Hochegg, betont, dass es auch eine Reihe jüngerer Patienten ohne jede Vorerkrankung gibt, die die SARS-CoV-2-Infektion besonders schwächt. „Post-COVID“ sei keine einheitliche Gruppe an Patienten, Beschwerden können unterschiedlich intensiv auftreten. Oft betroffen sind Personen, die im Spital beatmet werden mussten und von dort direkt zur Rehabilitation kommen. Diese sind körperlich stark geschwächt, und es ist ein Abbau ihrer Muskeln zu beobachten. Wie Lamprecht betont auch Winkler die Bedeutung der Atemmuskulatur, für die ebenso spezielle Trainings und physiotherapeutische Betreuung wichtig sind. Darauf bauen Maßnahmen auf, um die Gesamtkonstitution wiederherzustellen bzw. zu stärken.

Ebenso ein spezielles Programm entwickelte die Rehaklinik in Enns in Zusammenarbeit mit dem Kepler Universitätsklinikum: Die Post-COVID-Reha dauert drei Wochen. Physiotherapie, Kraft- und Ausdauertraining, Diätologie, Beratungen auch mit dem Angebot der Raucherentwöhnung und Entspannungsübungen tragen dazu bei, die Atemmuskulatur zu stärken und die Lungenfunktion wiederzuerlangen. Geachtet wird auch auf eine Behandlung des Erschöpfungssyndroms sowie von Konzentrationsstörungen oder Lähmungserscheinungen. Ergänzt wird dies mit Beratungen durch Sozialdienstmitarbeitende zu finanziellen Hilfen, wenn Corona-Patienten z. B. von Arbeitslosigkeit betroffen sind.

Im Rehabilitationszentrum Raxblick werden seit Juni 2020 Post-COVID-Patienten betreut, wobei die Nachfrage seit Anfang
2021 stark gestiegen ist. Bei Long COVID ist es oft sinnvoll, die 3-4-wöchige stationäre Rehabilitation durch eine ambulante Rehabilitation Phase 3 oder durch eine stationäre Folgerehabilitation nach 6 Monaten zu ergänzen, um eine weitere nachhaltige Verbesserung der Lebensqualität zu erzielen. „Insbesondere jüngere Patienten mit Long-COVID-Syndrom, die zuvor ein uneingeschränktes Leben führten, benötigen häufig auch eine intensive psychologische Betreuung“, sagt Stefan Kaltenegger, Ärztlicher Leiter der Pulmologischen Rehabilitation Raxblick.

Auch Einrichtungen wie die Therme Wien Med haben spezielle Programme für Patienten mit Long COVID entwickelt: Gearbeitet wird dort auch an neurologischen Beeinträchtigungen, Symptomen des Herz-Kreislauf-Systems und psychischen Belastungen. Angeboten wird ambulante Rehabilitation mit den Schwerpunkten Muskelaufbau, Atemtraining, Traumabewältigung, zu dem Patienten zwei- bis dreimal die Woche kommen und mehrere Stunden mit einem Trainingsprogramm verbrin-

\section{Roland Winkler, Hochegg: "Oft sind Patienten nach Beatmung geschwächt."}

gen. Dieses ist auf die jeweilige individuelle Situation ausgerichtet und beinhaltet Programmpunkte auch im angrenzenden Kurpark an der frischen Luft.

\footnotetext{
1 Nehme M et al (2020): COVID-19 Symptoms: Longitudinal Evolution and Persistence in Outpatient Settings. Anals of Internal Medicine. Zugang: https://www.acpjournals.org/doi/10.7326/M20-5926. Zugriff: 7.3.2021

2 Glöck R et al (2020): DGP-Empfehlungen zur pneumologischen Rehabilitation bei COVID-19. Zugang: https://www.ncbinlm.nih.gov/pmc/articles/PMC7516360/.Zugriff: 7.3.2021.

3 National Institute for Health and Excellence (NICE) (2020). COVID-19 rapid guideline: managing the long-term effects of COVID-19. Zugang: https://www.nice.org.uk/guidance/ng188/resources/COVID19rapid-guideline-managing-the-longterm-effects-of-COVID19-pdf-66142028400325. Zugriff: 7.3.2021.
}

\section{DR. CHRISTIAN F. FREISLEBEN}

freislebenagesundheitswirtschaft.at 